\title{
ARTIGOS
}

Submetido 29.07.2014. Aprovado 23.02.2015

Avaliado pelo processo de double blind review. Editora Científica: Ana Maria de Albuquerque Vasconcellos

DOI: http://dx.doi.org/10.1590/So034-759020150608

\section{ESTRATÉGIA SOCIOAMBIENTAL BASEADA EM RECURSOS E AMBIGUIDADE CAUSAL}

\author{
Resource-based social-environmental strategy and causal ambiguity \\ Estrategia socioambiental basada en recursos y ambiguedad causal
}

\section{RESUMO}

Este trabalho trata da influência da Estratégia Socioambiental Baseada em Recursos (RBSS [Resourcebased Socio-environmental Strategy]) na variável dependente ambiguidade causal. Esta, sob a ótica da Visão Baseada em Recursos da Firma (RBV [Resource-based View of the Firm]), funciona como um mecanismo de isolamento que impede a imitação pelos concorrentes. A RBSS foi operacionalizada a partir dos construtos orientação socioambiental, engajamento com stakeholders, aperfeiçoamento ambiental e capital humano. Os dados foram analisados com regressão linear. Como resultados, as hipóteses que trataram de orientação socioambiental e engajamento com stakeholders foram suportadas parcialmente, e aquelas que trataram de aperfeiçoamento ambiental e capital humano não foram suportadas.

PALAVRAS-CHAVE | Visão baseada em recursos da firma, estratégia socioambiental, estratégia socioambiental baseada em recursos, ambiguidade causal, capacidades organizacionais.

\begin{abstract}
This article discusses the influence of Resource-based Socio-environmental Strategy (RBSS) on causal ambiguity. From the perspective of the Resource-based View of the Firm (RBV), causal ambiguity works as an isolation mechanism that prevents imitation by competitors. The RBSS was operationalized by the following constructs: social orientation, stakeholder engagement, environmental improvement, and human capital. Data were analyzed using linear regression. We found that the hypotheses concerning Social Orientation and Stakeholder Engagement were partially supported, whereas the ones concerning Environmental Improvement and Human Capital were not.
\end{abstract}

KEYWORDS / Resource-based view of the firm, socio-environmental strategy, resource-based socioenvironmental strategy, causal ambiguity, organizational capabilities.

JOSÉ MILTON DE SOUSA-FILHO

miltonsousa@unifor.br

Professor da Universidade de

Fortaleza, Programa de Pós-

Graduação em Administração de

Empresas - Fortaleza - CE, Brasil

\section{JOSÉ CARLOS BARBIERI}

jose.barbieri@fgv.br

Professor da Fundação Getulio

Vargas, Escola de Administração de

Empresas de São Paulo - São Paulo - SP, Brasil

\section{RESUMEN}

Este trabajo trata de la influencia de la Estrategia Socio ambiental basada en Recursos (RBSS [Resource-based Socio-environmental Strategy]) en la variable dependiente ambigüedad causal. Esta, bajo la óptica de la Visión Basada en Recursos de la Firma (RBV [Resource-based View of the Firm]), funciona como un mecanismo de aislamiento que impide la imitación por parte de los competidores. La RBSS fue operacionalizada a partir de los constructos orientación socio ambiental, compromiso con stakeholders, perfeccionamiento ambiental y capital humano. Los datos fueron analizados con regresión lineal. Como resultados, las hipótesis que trataron de Orientación Socio ambiental y Compromiso con Stakeholders fueron soportadas parcialmente, y aquellas que trataron de Perfeccionamiento Ambiental y Capital Humano no fueron soportadas.

PALABRAS CLAVE / Visión basada en recursos de la firma, estrategia socio ambiental, estrategia socio ambiental basada en recursos, ambigüedad causal, capacidades organizacionales. 


\section{INTRODUÇÃO}

O presente trabalho trata do tema Estratégia Socioambiental Empresarial (ESAE). Ao analisar os trabalhos publicados no domínio dessa linha de pesquisa (Burke \& Logsdon, 1996; Husted, 2003; Husted \& Allen, 2007a, 2007b, 2009; Porter \& Kramer, 2002, 2006, 2011), o principal mote tem sido o relacionamento entre a Responsabilidade Socioambiental Empresarial (RSAE) e a geração de vantagens competitivas, sendo esse um dos principais construtos do campo da estratégia (Powell, 2001). Para tentar explicar a vantagem competitiva trazida pelas ações de RSAE, a Visão Baseada em Recursos da Firma (RBV[Resource-based View of the Firm]) tem regularmente sido utilizada (Aragón-Correa \& Sharma, 2003; Branco \& Rodrigues, 2006; Hart, 1995; Hart \& Dowell, 2011; McWilliams \& Siegel, 2001, 2011; Russo \& Fouts, 1997; Sharma \& Vredenburg, 1998) e, com isso, tem se tornado a mais influente teoria sobre vantagem competitiva no campo da ESAE (Walls, Phan, \& Berrone, 2011).

Na perspectiva da RBV, a vantagem competitiva é uma função da combinação de recursos valiosos e raros, e a sustentação da vantagem competitiva é atingida a partir da combinação de recursos inimitáveis e insubstituíveis (Barney, 1991). Alguns autores (King, 2007; King \& Zeithaml, 2001; Mosakowski, 1997; Simonin, 1999) argumentam que o aspecto da inimitabilidade é a principal contribuição da RBV, já que recursos difíceis de imitar podem criar mecanismos de isolamento que ajudam a empresa a sustentar sua vantagem competitiva.

Se, por um lado, o foco dos estudos que relacionam RSAE e RBV tem sido a relação entre RSAE e a geração de vantagens competitivas, por outro, não se tem estudado a relação entre ESAE e um dos drivers que levam à vantagem competitiva, nesse caso, a ambiguidade causal. Sob tal aspecto, pode-se constatar uma lacuna teórico-empírica, já que não foi encontrado nenhum estudo abordando a relação entre a ESAE baseada em recursos e a criação de ambiguidade causal. Com isso, propõe-se a pergunta de pesquisa que norteia o presente trabalho: Qual a relação entre estratégia socioambiental baseada em recursos e ambiguidade causal?

A delimitação teórica desta pesquisa enfoca ESAE baseada em recursos e seu impacto na criação de ambiguidade causal. Vale ressaltar que os construtos vantagem competitiva e desempenho financeiro, apesar de importantes, não fazem parte do escopo deste trabalho. Assim, o objetivo geral é investigar a relação entre estratégia socioambiental baseada em recursos e ambiguidade causal. 0 presente trabalho está estruturado em cinco partes. Em seguida, estão apresentadas a teoria e hipóteses, metodologia, resultados, e discussão e conclusões.

\section{Teoria e hipóteses}

Os recursos da firma podem ser difíceis de imitar por três razões (Barney, 1991): (a) a habilidade de obter ou desenvolver recursos depende de condições históricas relacionadas à firma (path dependence) (Barney, 1991); (b) a ligação entre os recursos que a firma possui e a sustentação de sua vantagem competitiva é causalmente ambígua (Barney, 1991; Reed \& DeFillippi, 1990), e (c) o recurso que gera a vantagem para a firma é socialmente complexo (Barney, 1991; Dierickx \& Cool, 1989). Essas três características têm sido chamadas na literatura de "mecanismos de isolamento" (Mahoney \& Pandian, 1992; Rumelt, 1984) e originam a fonte da dificuldade de imitação, que, por consequência, sustenta a vantagem competitiva da firma.

Reed e DeFillippi (1990) afirmam que as barreiras mais efetivas contra imitação são alcançadas quando os concorrentes não compreendem as competências nas quais a vantagem está baseada. As competências às quais Reed e DeFillippi (1990) se referem são chamadas pelos próprios autores de "causalmente ambíguas”, ou seja, são recursos combinados que geram ambiguidade causal. Segundo Barney (1991), existe ambiguidade causal quando a ligação entre os recursos controlados pela firma e sua vantagem competitiva sustentável não pode ser entendida, ou é entendida muito imperfeitamente. Quando a ligação entre os recursos da firma e a vantagem competitiva sustentável é insuficientemente entendida, torna-se difícil para os concorrentes imitarem as estratégias de uma empresa bem-sucedida, já que não conseguem saber quais recursos devem imitar (Barney, 1991). Nessa linha, Peteraf (1993, p. 182) afirma que um dos fatores fundamentais que colaboram para a dificuldade de imitação é a ambiguidade causal, pois previne que aqueles concorrentes dispostos a imitar saibam exatamente o que imitar ou como imitar a fonte da vantagem.

De acordo com Reed e DeFillippi (1990), existem três características as quais os recursos e competências devem possuir, individualmente ou em conjunto, para gerar ambiguidade causal. São elas tacitividade (Polanyi, 1967), complexidade (Nelson \& Winter, 1982) e especificidade (Williamson, 1985). 0 impacto dos diversos tipos de recursos e competências que possuem essas três características (tacitividade, complexidade e especificidade) sobre a ambiguidade causal ainda precisam ser mais aprofundados de maneira teórica e empírica. Neste trabalho, o foco escolhido para aprofundamento foi a influência da estratégia socioambiental baseada em recursos sobre ambiguidade causal.

Dessa forma, neste trabalho, a ambiguidade causal será tratada como variável dependente, em contraponto aos trabaIhos anteriores onde esta foi tratada como variável moderadora (Ambrosini \& Bowman, 2005; González-Alvarez \& Nieto-Anto- 
lín, 2005; Powell, Lovallo, \& Caringal, 2006; Simonin, 1999). As capacidades baseadas em recursos socioambientais que serão descritas a seguir possuem as características causalmente ambíguas preconizadas por Reed e DeFillippi (1990): tacitividade, complexidade e especificidade. Conforme argumentado anteriormente, tais características são essenciais para que o recurso ou capacidade tenha o potencial de gerar ambiguidade causal. Assim, os seguintes recursos e capacidades emergiram do campo teórico: (a) orientação socioambiental, (b) engajamento com stakeholders, (c) aperfeiçoamento ambiental, e (d) capital humano.

\section{Orientação socioambiental}

Orientação socioambiental é o comportamento positivo da empresa no que diz respeito a questões sociais, ambientais e éticas, vinculadas ao modo de fazer negócios e aos valores da organização. A orientação socioambiental emerge como capacidade em diversos trabalhos e, por vezes, com nomenclaturas diferentes. Husted e Allen (2007a), a partir da discussão sobre ideologia e valores organizacionais, introduzem o termo social responsibility orientation, afirmando que tal orientação se refere ao comprometimento da empresa em participar das soluções para os problemas sociais. Segundo os autores, são os valores que definirão, e suportarão, o comprometimento da empresa com as causas e ações sociais; e um forte comprometimento com a responsabilidade social fornece um conjunto de valores que são difíceis de copiar pelos concorrentes (Barney, 1986; Husted \& Allen, 2007a).

A orientação socioambiental também emerge na pesquisa de Walls, Phan, e Berrone (2011), com o termo historical orientation. Esse termo, na pesquisa de Walls et al. (2011), refere-se à orientação socioambiental da firma ao longo do tempo, e com isso pode-se observar o padrão de tomadas de decisão em prol de questões socioambientais em diferentes momentos da existência da empresa. Segundo Walls et al. (2011), quanto maior a história da firma em relação à sua orientação socioambiental, maior será a chance de ela ter construído capacidades e recursos ambientais idiossincráticos, e, consequentemente, difíceis de copiar.

De acordo com Hart (1995) e Russo e Fouts (1997), a estratégia socioambiental é refletida nos valores da empresa e também dos gestores, que, em seguida, são integrados aos sistemas de gestão e à estratégia do negócio. Sharfman, Pinkston, e Sigerstad (2000) indicam que os valores atuam como uma moldura no que diz respeito à avaliação e reconhecimento de quais ações sociais ou ambientais a empresa deve adotar. Assim, essas interpretações organizacionais e gerenciais em relação às questões socioambientais afetam diretamente a seleção e implementação das estratégias socioambientais (Bansal \& Roth, 2000; Husted \& Allen, 2007a; Sharma, 2000; Sharma et al., 1999).

Além disso, aspectos como ética (Black, 2006; Husted \& Allen, 2000) e governança (Walls, Berrone, \& Phan, 2012; Post, Rahman, \& Rubow, 2011) fazem parte da orientação socioambiental de qualquer organização. Walls et al. (2012), por exemplo, estudaram a relação entre o conselho de administração e a adoção de estratégias socioambientais, encontrado relação positiva quando os membros do conselho tinham clara propensão e orientação voltada a apoiar questões sociais e ambientais.

Assim, conforme pode ser percebido, aspectos como dependência da história, ética e governança fazem parte da capacidade orientação socioambiental. Todos esses aspectos são intangíveis e têm características complexas, que emergem a partir do conhecimento tácito e da história das organizações. Dessa forma, pode-se considerá-las causalmente ambíguas, sendo difícil para os concorrentes entender de maneira clara como impactam o dia a dia e a estratégia de longo prazo do negócio.

Em conclusão, é válido o argumento de que uma estratégia socioambiental baseada na capacidade de orientação socioambiental, devido a suas características causalmente ambíguas, tem potencial para criar ambiguidade causal. Assim, deriva-se a Hipótese 1.

$\mathrm{H}_{1}$ : A estratégia socioambiental baseada na capacidade orientação socioambiental impacta de maneira positiva a criação de ambiguidade causal.

\section{Engajamento com stakeholders}

A discussão sobre o engajamento com stakeholders está presente nos textos sobre estratégia socioambiental desde Hart (1995). Essa é provavelmente a capacidade mais citada nos diversos artigos sobre o tema, principalmente naqueles que utilizam a perspectiva da RBV (Black, 2006; Husted \& Allen, 2007a, 2007b, 2009; Sharma \& Vredenburg, 1998; Walls et al., 2011). Segundo Donaldson e Preston (1995), a empresa orientada para o relacionamento com os stakeholders tende a ter seus interesses legitimados com mais facilidade; além disso, tal relação mútua gera uma possível troca de benefícios. Para Atkinson, Waterhouse, e Wells (1997), a organização moderna é uma rede de contratos implícitos e explícitos que especifica a relação entre a empresa e seus stakeholders.

Molteni (2006) e Black (2006) corroboram o raciocínio de Hart (1995) e Sharma e Vredenburg (1998) no que diz respeito à complexidade do processo de relacionamento entre empresas e seus públicos de interesse. 0 fato de a empresa precisar entender necessidades específicas dos stakeholders, e, com isso, obter 
ganhos ainda mais específicos, torna esse processo difícil de ser compreendido e, consequentemente, imitado pelos concorrentes devido a sua especificidade. Segundo Sharma e Henriques (2005), em resposta à influência dos stakeholders, as empresas podem desenvolver estratégias socioambientais específicas como o ecodesign ou a gestão do ecossistema em que estão inseridas.

0 recurso relacionamento foi estudado por Dyer e Singh (1998), que fizeram a proposição da visão relacional (relational view). Nesta, a vantagem obtida a partir do relacionamento pode vir de três aspectos, sendo eles (a) acesso a ativos específicos a partir do relacionamento, (b) compartilhamento de rotinas e conhecimentos, e (c) complementaridade de recursos e capacidades. 0 fato de um stakeholder específico apoiar o desenvolvimento de um produto, ou ajudar a melhorar o relacionamento da firma com seus clientes, torna o relacionamento vantajoso para a empresa.

O relacionamento e as interações entre empresas e seus públicos de interesse envolvem recursos idiossincráticos que são muito difíceis de serem copiados, devido a sua intangibilidade. Além disso, o relacionamento da empresa com cada um dos stakeholders é diferente e envolve ativos específicos, o que fornece um grau ainda maior de complexidade caso um concorrente queira imitar. Dessa forma, pode-se perceber que o engajamento com stakeholders é uma capacidade que tem características causalmente ambíguas, pois envolve complexidade e especificidade.

Em conclusão, é válido o argumento de que uma estratégia socioambiental baseada na capacidade de engajamento com stakeholders, devido a suas características causalmente ambíguas, tem potencial para criar ambiguidade causal. Assim, deriva-se a Hipótese 2.

H2: A estratégia socioambiental baseada na capacidade engajamento com stakeholders impacta de maneira positiva a criação de ambiguidade causal.

\section{Aperfeiçoamento ambiental}

No contexto dos artigos que trataram da estratégia socioambiental baseada em recursos, expressões como melhoria contínua (Hart, 1995), melhorias operacionais (Shrivastava, 1995) e inovação contínua (Sharma \& Vredenburg, 1998) são diferentes termos para o mesmo fenômeno, a capacidade de aperfeiçoar as operações com foco no meio ambiente. Aliado a isso, a participação em redes de suprimentos (Walls et al., 2011) e a colaboração na cadeia de suprimentos (Klassen \& Vachon, 2003), com orientação às questões socioambientais, também são atividades que ajudam a suportar o processo de melhoria contínua. A combinação desses recursos, que enfocam a operação e a cadeia de suprimentos, cria a capacidade de aperfeiçoamento ambiental (enviromental improvement).

O aperfeiçoamento ambiental é a capacidade da empresa de melhorar os aspectos ambientais de suas operações implementando técnicas, tecnologias ou metodologias que gerem impactos positivos nos desempenhos financeiro e socioambiental. De acordo com Shrivastava (1995), tecnologias ambientais são potenciais recursos estratégicos porque afetam a cadeia de valor em múltiplos pontos. Dessa forma, essas tecnologias são capazes de fornecer às empresas vantagens únicas e inimitáveis em cada estágio da cadeia de valor (Shrivastava, 1995).

O aperfeiçoamento das operações enfocando questões ambientais como prevenção da poluição (Aragón-Correa \& Sharma, 2003; Hart, 1995; Hart \& Dowell, 2011; Russo \& Fouts, 1997), implementação de novas tecnologias ambientais (Shrivastava, 1995), colaboração na cadeia de suprimentos para redução dos impactos ambientais (Klassen \& Vachon, 2003), orientação em busca da cadeia de suprimentos sustentável (Kleindorfer, Singhal, \& Wassenhove, 2005; Linton, Klassen, \& Jayaraman, 2007), ecodesign (Sarkis, 1995), tecnologias limpas e produtividade dos recursos (Hart \& Dowell, 2011; Porter \& Van der Linde, 1995), extensão de vida útil de produto (Linton \& Jayaraman, 2005), sistema de gestão ambiental (Kitazawa \& Sarkis, 2000; Melnyk, Sroufe, \& Calantone, 2003; Sroufe, 2003), entre outras, utiliza uma grande quantidade de ativos combinados.

Conforme afirmaram Russo e Fouts (1997) e Aragón-Correa e Sharma (2003), a prevenção da poluição, por exemplo, é um processo socialmente complexo e que necessita do envolvimento de funcionários de diferentes áreas, além da integração e coordenação desses recursos. Walls et al. (2011) argumentam que a estratégia socioambiental proativa enfatiza a prevenção e esta só é capaz disso a partir de uma combinação de recursos única, por meio da qual as firmas desenvolvem capacidades socioambientais.

No âmbito da capacidade de aperfeiçoamento ambiental, é indispensável a integração entre recursos humanos, operação e tecnologia, tudo isso sendo influenciado pelas idiossincrasias da empresa. Assim, essa capacidade tem características causalmente ambíguas, como tacitividade, complexidade e especificidade. Em conclusão, é válido o argumento de que uma estratégia socioambiental baseada na capacidade de aperfeiçoamento ambiental, devido a suas características causalmente ambíguas, tem potencial para criar ambiguidade causal. Assim, deriva-se a Hipótese 3.

H3: A estratégia socioambiental baseada na capacidade aperfeiçoamento ambiental impacta de maneira positiva a criação de ambiguidade causal. 


\section{Capital humano}

Hart (1995) e McWilliams e Siegel (2011) consideram o capital humano (funcionários, incluindo a alta gestão) um recurso estratégico à luz da RBV. Segundo McWilliams e Siegel (2011), os funcionários podem ser um recurso estratégico quando são mais produtivos que os funcionários dos concorrentes, e o resultado disso pode ser visto na facilidade em recrutar novos talentos e na motivação da equipe. Ainda segundo os autores, as práticas de RSAE têm assumido um papel importante nos processos de recursos humanos, inclusive na função de tornar os funcionários mais produtivos. Segundo Branco e Rodrigues (2006), investir em ações de RSAE gera um impacto importante na criação de recursos intangíveis fundamentais associados aos recursos humanos.

A literatura já explorou a relação teórica estabelecida entre a RSAE e o desenvolvimento de capital humano, sendo esse último considerado um recurso organizacional intangivel. Conforme McWilliams e Siegel (2011), o estudo de Nyborg e Brekke (2004) afirma que a execução de atividades de RSAE permite à empresa desenvolver capacidades de capital humano, como a possibilidade de atrair funcionários altamente motivados e produtivos. De acordo com Surroca, Tribó, e Waddock (2010), a RSAE contribui para o desenvolvimento do capital humano em três aspectos: (a) atração e retenção de talentos, (b) influência positiva sobre as atitudes dos funcionários, e (c) práticas de gestão de recursos humanos que encorajam os funcionários a envolver-se com questões socioambientais. A inserção de aspectos socioambientais na gestão das pessoas pode ampliar o desenvolvimento e a acumulação desse recurso.

A acumulação de capital humano derivada de práticas socioambientais pode tornar-se fonte de vantagem competitiva e, com isso, melhorar o desempenho da organização (Becker \& Gerhart, 1996; Huselid, 1995; Oliveira \& Oliveira, 2011; Pfeffer \& Veiga, 1999). Hart e Milstein (2003) corroboram essa ideia afirmando que o desempenho ambiental afeta o desempenho geral da organização por meio do desenvolvimento de novos recursos intangíveis relacionados aos recursos humanos. Por exemplo, Hart e Milstein (2003) argumentam que a efetiva prevenção da poluição requer um intensivo envolvimento das pessoas, bem como o desenvolvimento de capacidades no âmbito da melhoria contínua e gestão da qualidade ambiental.

Hanna, Newman, e Johnson (2000) estudaram o envolvimento de 349 funcionários em projetos ambientais e seu impacto no desempenho ambiental e financeiro de empresas manufatureiras. Como resultado, os autores encontraram forte relação positiva entre as metas operacionais, o envolvimento dos times de projeto e os resultados ambientais. 0 artigo sugere, ainda, a importância dos esforços de melhoria contínua dos gestores de operação, e entre esses esforços está a orientação ao envolvimento das pessoas com a gestão ambiental, que pode ser um recurso-chave na melhoria dos resultados ambientais.

Na pesquisa de Walls et al. (2011), entre as seis categorias vinculadas à estratégia socioambiental encontradas, três dizem respeito ao desenvolvimento de pessoas, são elas: (a) visão gerencial, (b) habilidades da alta gestão, e (c) sistemas de recursos humanos. Ao observar tais categorias, pode-se notar que abordam aspectos intangíveis da organização. A visão gerencial e as habilidades da alta gestão, por exemplo, são recursos intangíveis com grande carga de imaterialidade, difíceis de serem compreendidos pelos concorrentes; além disso, sofrem influências das idiossincrasias organizacionais, da cultura e do background dos gestores. Os sistemas de recursos humanos também sofrem as mesmas influências, e são ainda impactados pela visão gerencial.

Conforme pode-se perceber, a relação entre a estratégia socioambiental e o capital humano é permeada por influências e recursos com características causalmente ambíguas. Em conclusão, é válido o argumento de que uma estratégia socioambiental baseada na capacidade de desenvolver o capital humano em prol de uma orientação socioambiental, devido a suas características causalmente ambíguas, tem potencial para criar ambiguidade causal. Assim, deriva-se a Hipótese 4.

H4: A estratégia socioambiental baseada na capacidade capital humano impacta de maneira positiva a criação de ambiguidade causal.

\section{METODOLOGIA}

A metodologia adotada possui um caráter exploratório-descritivo. 0 estudo exploratório serve de base para a formulação das hipóteses, isolando variáveis e relações-chave para análises descritivas (Cooper \& Schindler, 2011; Hair, Babin, Money, \& Samouel, 2005). Durante a fase inicial exploratória, foram executadas: (a) a revisão da literatura, (b) a definição dos construtos, e (c) o desenvolvimento das hipóteses. A fase descritiva contou com (d) o levantamento dos dados necessários para testar as hipóteses e (e) com a preparação do banco de dados, onde foram combinados os dados de cada empresa retirados de uma e de outra base, além da criação de variáveis dummy necessárias à análise.

Os dados utilizados nesta pesquisa foram coletados em duas bases de dados diferentes. Aqueles referentes à estratégia socioambiental baseada em recursos foram coletados na base Sustainalytics Global Platform, e os dados referentes ao construto ambiguidade causal foram coletados na Compustat Global. Segundo Walls et al. (2011), coletar dados em bases diferentes é interessante no intuito de reduzir o potencial viés de uma única 
base de dados. No total, a amostra contou com 245 empresas da Europa, América do Norte e Ásia-Pacífico, conforme disponibilidade da Sustainalytics Global Platform.

A Sustainalytics fornece um total de 11 indicadores que são calculados a partir de um conjunto de 70 itens (neste trabalho, foram utilizados apenas 8 dos 11 indicadores), que podem ser vistos no Modelo 1. Não foram encontradas na literatura proxies para ambiguidade causal (AMBC), assim optou-se por utilizar o grau de intangibilidade (Kayo \& Famá, 2004) que é calculado a partir da divisão do valor de mercado pelo patrimônio líquido contábil.

Os dados foram coletados entre agosto e outubro de 2012. Contudo, os dados utilizados nesta pesquisa (tanto da Sustainalytics como da Compustat) são referentes ao ano de 2009. Isso ocorreu devido à disponibilidade de dados da Sustainalytics, já que a base fornecida não continha dados nem anteriores, nem posteriores a 2009, sendo este o único ano disponível. 0 fato de utilizar dados de apenas um ano não prejudica a pesquisa, já que esta não se propõe a ser uma pesquisa longitudinal, e, além disso, outros pesquisadores já utilizaram esse formato de pesquisa, como Griffin e Mahon (1997).

Para testar as hipóteses do estudo, foi formulado o modelo (equação de regressão) para análise de regressão múltipla a seguir, que trata da influência da Estratégia Socioambiental Baseada em Recursos na variável dependente ambiguidade causal.

$A M B C=\beta_{0}+\beta_{1} B U S E T H+\beta_{2} C O R P G O V+$

$\beta_{3}$ SOCCOMM $+\beta_{4}$ SOCSUP $+\beta_{5} P U B P O L+\beta_{6} O P E R+$

(Modelo 1)

$\beta_{7} E N V S U P+\beta_{8} E M P+\beta_{9} I N D+\beta_{10} \operatorname{LogFUNC}+\varepsilon$

Onde:

$A M B C=$ Proxy para ambiguidade causal (logaritmo base 10 do grau de intangibilidade)

BUSETH = Ética nos negócios (orientação socioambiental) CORPGOV = Governança corporativa (orientação socioambiental)

SOCCOMM = Sociedade e comunidade (engajamento com stakeholders)

SOCSUP = Relacionamento social com fornecedores (engajamento com stakeholders)

PUBPOL = Política pública (engajamento com stakeholders) OPER = Operações (aperfeiçoamento ambiental)

ENVSUP = Relacionamento ambiental na cadeia de suprimentos (aperfeiçoamento ambiental)

$E M P=$ Tratamento dos colaboradores (capital humano)

IND = Indústria (dummy)

LogFUNC = Logaritmo (base 10) da quantidade de funcionários

A seguir, serão apresentados os resultados da pesquisa.

\section{RESULTADOS}

Inicialmente, procedeu-se a análise de confiabilidade do construto utilizado no estudo, formado pelas dimensões escolhidas na base Sustainalytics. A verificação foi realizada por meio do Alpha de Cronbach. A análise do construto Estratégia Socioambiental Baseada em Recursos, com suas oito variáveis, retornou um Alpha de 0,726 , e, ao considerar o cálculo com os itens padronizados, 0 Alpha aumentou para 0,733. O valor de Alpha recomendando por Hair, Black, Babin, Anderson, e Tatham (2009) é de no mínimo 0,700 , sendo que, para estudos exploratórios, que é o caso desta pesquisa, esses autores afirmam que um Alpha acima de 0,600 já seria aceitável. Dessa forma, pode-se observar que o construto utilizado é confiável nos padrões que a literatura recomenda. A Tabela 1 apresenta o resultado do teste Alpha de Cronbach.

\section{Tabela 1. Alpha de Cronbach}

\begin{tabular}{|c|c|c|c|}
\hline \multicolumn{4}{|c|}{ Confiabilidade estatística } \\
\hline \multicolumn{2}{|c|}{ Alpha de Cronbach } & $\begin{array}{c}\text { Alpha de Cronbach baseado } \\
\text { em itens padronizados }\end{array}$ & $\begin{array}{l}\text { No. de } \\
\text { itens }\end{array}$ \\
\hline \multicolumn{2}{|l|}{0,726} & 0,733 & 8 \\
\hline \multicolumn{4}{|c|}{ Casos } \\
\hline & & $\mathrm{N}$ & $\%$ \\
\hline \multirow{3}{*}{ Cases } & Válidos & 245 & 100,0 \\
\hline & Excluídos & $\mathrm{O}$ & 0 \\
\hline & Total & 245 & 100,0 \\
\hline
\end{tabular}

Em seguida, conduziu-se a análise de regressão linear múltipla com o objetivo de testar as hipóteses da pesquisa. 0 modelo de regressão apresentou um coeficiente de determinação $\left(R^{2}\right)$ de $65,4 \%(0,654)$ e um coeficiente de determinação ajustado ( $R^{2}$ Ajustado) de 61,6\% (o,616). Dessa forma, pode-se afirmar que as variáveis independentes utilizadas explicam 61,6\% da variação da variável dependente, já que o modelo como um todo é significante $(0,000)$. 0 erro padrão da estimativa foi de 0,718 , adequado para a estatística. Além disso, pode-se observar a ausência de autocorrelação serial (um dos pressupostos da análise de regressão) que pode ser avaliada por meio do teste de Durbin-Watson. Esse teste mostra que os resíduos da regressão são independentes, ou seja, não são correlacionados. A Tabela 2 mostra o valor de 1,934 para esse teste. Conforme Corrar, Paulo e Dias-Filho (2009), os valores adequados desse teste devem ser abaixo de 2,00, e, dessa forma, pode-se constatar que o modelo não apresenta problemas de autocorrelação serial. 
Tabela 2. Resultado do modelo de regressão múltipla $\left(\mathbf{R}^{2}\right)$

\begin{tabular}{|c|c|c|c|c|c|c|c|c|c|c|}
\hline \multirow[b]{2}{*}{ Modelo } & \multirow[b]{2}{*}{$\mathbf{R}$} & \multirow[b]{2}{*}{$\mathrm{R}^{2}$} & \multirow[b]{2}{*}{$R^{2}$ ajustado } & \multirow{2}{*}{$\begin{array}{c}\text { Erro } \\
\text { padrão }\end{array}$} & \multicolumn{5}{|c|}{ Estatística de mudança } & \multirow{2}{*}{$\begin{array}{l}\text { Durbin- } \\
\text { Watson }\end{array}$} \\
\hline & & & & & $\begin{array}{c}\text { Mudança } \\
\text { no } R^{2}\end{array}$ & $\begin{array}{c}\text { Mudança } \\
\text { em F }\end{array}$ & $\mathbf{g l}^{1}$ & $\mathrm{gl}^{2}$ & $\begin{array}{c}\text { Mudança de } \\
\text { significância } \\
\text { em F }\end{array}$ & \\
\hline 1 & $0,809^{a}$ & 0,654 & 0,616 & 0,71809 & 0,654 & 17,242 & 24 & 219 & 0,000 & 1,934 \\
\hline
\end{tabular}

a. Preditores: (Constant), América do Norte, política pública, containers e acondicionamento, papel e celulose, desenvolvimento de produtos, materiais de construção, distribuição e trading, conglomerados industriais, aeroespacial e defesa, equipamentos elétricos, construção e engenharia, capital humano, equipamentos e serviços de energia, ética nos negócios, óleo, gás e combustíveis, log num. funcionários, maquinaria, Europa, operações, sociedade e comunidade, relacionamento social com fornecedores, governança corporativa, relacionamento ambiental na cadeia de suprimentos, metais e mineração

b. Variável dependente: Ambiguidade causal

A análise de regressão apresentou a soma dos quadrados no valor de 326,310, com resíduos no valor de 112,928. A soma dos quadrados é o resíduo quadrado caso fosse utilizada apenas a média da variável dependente ambiguidade causal para predição. Ao incluir as variáveis independentes na equação, esse resíduo caiu para 112,928, ou seja, melhorou o poder de predição do modelo. 0 teste ANOVA ( $F=17,242$, Sig < 0,001) com significância estatística atesta que o modelo é válido, pois rejeita-se a hipótese de que o $\mathrm{R}^{2}$ é igual a zero. Tais resultados podem ser vistos na Tabela 3.

Tabela 3. Teste ANOVA do modelo de regressão múltipla

\begin{tabular}{|c|c|c|c|c|c|c|}
\hline \multicolumn{7}{|c|}{ ANOVA $^{a}$} \\
\hline \multicolumn{2}{|r|}{ Modelo } & Soma de & GI & Quadrado & $F$ & Sig. \\
\hline \multirow{3}{*}{1} & Regressão & 213,382 & 24 & 8,891 & 17,242 & $0,000^{b}$ \\
\hline & Resíduos & 112,928 & 219 & 0,516 & & \\
\hline & Total & 326,310 & 243 & & & \\
\hline
\end{tabular}

a. Variável dependente: Ambiguidade causal

b. Preditores: (Constant), América do Norte, política pública, containers e acondicionamento, papel e celulose, desenvolvimento de produtos, materiais de construção, distribuição e trading, conglomerados industriais, aeroespacial e defesa, equipamentos elétricos, construção e engenharia, capital humano, equipamentos e serviços de energia, ética nos negócios, óleo, gás e combustíveis, log num. funcionários, maquinaria, Europa, operações, sociedade e comunidade, relacionamento social com fornecedores, governança corporativa, relacionamento ambiental na cadeia de suprimentos, metais e mineração

A partir dos resultados da análise de regressão, pôde-se observar que somente algumas variáveis apresentaram signifi- cância estatística (Tabela 4), assim, estas constituíram a seguinte equação de regressão:

$\mathrm{AMBC}=0,024$ CORPGOV $+0,008$ SOCCOMM - 0,025 OPER - 0,009 ENVSUP + 0,894 AERDEF + 0,583 EQSENR + 0,521 MAQ + 1,214 EURO + 1,151 AMERN

Onde:

$\mathrm{AMBC}=$ Proxy para ambiguidade causal (logaritmo do grau de intangibilidade)

CORPGOV = Governança corporativa (orientação socioambiental)

SOCCOMM = Sociedade e comunidade (engajamento com stakeholders)

OPER = Operações (aperfeiçoamento ambiental)

ENVSUP = Relacionamento ambiental na cadeia de suprimentos (aperfeiçoamento ambiental)

$A E R D E F=$ Indústria aeroespacial e defesa

EQSENR = Indústria equipamentos e serviços de energia

$M A Q=$ Indústria maquinária

EURO = Região Europa

AMERN = Região América do Norte

A Hipótese $1\left(\mathrm{H}_{1}\right)$ trata do impacto do construto orientação socioambiental na variável dependente ambiguidade causal, 
cujo enunciado é "a estratégia socioambiental baseada na capacidade orientação socioambiental impacta de maneira positiva a criação de ambiguidade causal”. As variáveis que representaram a orientação socioambiental foram ética nos negócios e governança corporativa. A variável Ética nos Negócios ( $t=-0,304, p$ = 0,709) não impactou a variável dependente, já que seu coeficiente não se apresentou significativamente diferente de zero. Por outro lado, a variável governança corporativa $(t=5,201, p$ = 0,001) tem significância estatística e coeficiente padronizado Beta de 0,304, apesar do coeficiente de regressão muito baixo, 0,024 . Dessa forma, pode-se afirmar que $\mathrm{H}_{1}$ foi suportada parcialmente, pois uma de suas variáveis, governança corporativa, influencia a variável dependente.

A Hipótese $2\left(\mathrm{H}_{2}\right)$ trata do impacto do construto engajamento com stakeholders na variável dependente ambiguidade causal. Entre as variáveis que representaram o engajamento com stakeholders, somente sociedade e comunidade $(t=2,247$, $p=0,026)$ obteve significância estatística, e possui coeficiente padronizado beta de 0,122 (e coeficiente de regressão muito baixo, o,008). As outras variáveis do construto, relacionamento social com fornecedores ( $t=0,593, p=0,554)$ e política pública $(t=0,174, p=0,862)$, não alcançaram significância estatística. Apesar do baixo nível do coeficiente beta $(0,122)$ da variável sociedade e comunidade, pode-se afirmar que $\mathrm{H}_{2}$ foi suportada parcialmente, pois essa variável explica parte do fenômeno.

A Hipótese $3\left(\mathrm{H}_{3}\right)$ trata do impacto do construto aperfeiçoamento ambiental sobre a variável dependente ambiguidade causal. As variáveis que representaram o construto apresentaram significância estatística, porém exibiram comportamento diferente daquele proposto na hipótese. A variável operações (t $=-4,673, p=0,001)$ tem coeficiente padronizado beta com sinal negativo $(-0,257)$, bem como a variável relacionamento ambiental na cadeia de suprimentos $(t=-2,359, p=0,019)$, que também possui sinal negativo em seu coeficiente beta $(-0,143)$. Dessa forma, pode-se afirmar que $\mathrm{H}_{3}$ não foi suportada, já que o sinal negativo do coeficiente representa um impacto negativo na variável dependente, resultado contrário ao que propôs a hipótese.

A Hipótese $4\left(\mathrm{H}_{4}\right)$ trata do impacto do construto capital humano sobre a variável dependente ambiguidade causal. A única variável que representou o construto não obteve significância estatística $(t=0,369, p=0,713)$, dessa forma, pode-se afirmar que $\mathrm{H}_{4}$ não foi suportada.

Ao observar as variáveis de controle, pôde-se constatar que o tamanho da empresa (calculado pelo logaritmo da quantidade de funcionários) não influenciou a regressão, já que não obteve coeficiente significante $(t=-0,115, p=0,909)$; isso provavelmente ocorreu devido à similaridade entre o tamanho das empresas, todas de capital aberto e com grande quantidade de funcionários. No entanto, pode-se verificar que as indústrias aeroespacial e defesa $(t=3,076, p=0,002)$, equipamentos e serviços de energia $(t=2,190, p=0,030)$, e maquinaria $(t=2,774$, $p=0,006)$, bem como as regiões Europa $(t=9,624, p=0,000)$ e América do Norte $(t=3,232, p=0,001)$, apresentaram significância estatística. Entre as variáveis de controle, 02 (duas) foram automaticamente excluídas da análise pelo software, indústria química e região Ásia-Pacífico. Todos os coeficientes de regressão (B), coeficientes padronizados beta, e a significância estatística de cada variável, podem ser observados na Tabela 4

Tabela 4. Coeficientes significantes do modelo de regressão múltipla

\begin{tabular}{|c|c|c|c|c|c|c|c|c|}
\hline \multirow{2}{*}{\multicolumn{2}{|c|}{ Modelo }} & \multirow{2}{*}{$\begin{array}{c}\text { Coeficientes } \\
\text { B }\end{array}$} & \multirow{2}{*}{$\begin{array}{c}\text { Erro } \\
\text { padrão }\end{array}$} & \multirow{2}{*}{$\begin{array}{l}\text { Coeficientes } \\
\text { padronizados } \\
\text { Beta }\end{array}$} & \multirow{2}{*}{$\mathrm{t}$} & \multirow{2}{*}{ Sig. } & \multicolumn{2}{|c|}{ Colinearidade } \\
\hline & & & & & & & Tolerância & VIF \\
\hline \multirow{6}{*}{1} & (Constant) & 0,476 & 0,693 & & 0,687 & 0,493 & & \\
\hline & Governança corporativa & 0,024 & 0,005 & 0,304 & 5,201 & 0,000 & 0,462 & 2,166 \\
\hline & Sociedade e comunidade & 0,008 & 0,004 & 0,122 & 2,247 & 0,026 & 0,536 & 1,866 \\
\hline & $\begin{array}{l}\text { Relacionamento ambiental na cadeia } \\
\text { de suprimentos }\end{array}$ & $-0,009$ & 0,004 & $-0,143$ & $-2,359$ & 0,019 & 0,431 & 2,322 \\
\hline & Aeroespacial e defesa & 0,894 & 0,291 & 0,153 & 3,076 & 0,002 & 0,637 & 1,570 \\
\hline & Equipamentos e serviços de energia & 0,583 & 0,266 & 0,113 & 2,190 & 0,030 & 0,591 & 1,691 \\
\hline
\end{tabular}

a. Variável dependente: Ambiguidade causal 
Em seguida, foi realizada a análise dos pressupostos da regressão, no intuito de verificar a integridade dos testes de ajustamento e de significância do modelo. A ausência de multicolinearidade pode ser comprovada pela estatística de colinearidade, especificamente pelo teste VIF. Conforme pode-se constatar na última coluna da Tabela 4, o VIF de cada variável variou entre 1,110 e 2,449. Hair et al. (2009) recomendam que - VIF adequado deve ficar abaixo de 10, dessa forma, percebe-se que não existe problema de multicolinearidade no modelo.

Para verificar a normalidade dos resíduos, procedeu-se com o teste não paramétrico Kolmogorov-Smirnov (KS). Esse teste examina se a série de dados está conforme a distribuição esperada, nesse caso, a distribuição normal. A hipótese nula é que a distribuição da série de dados é normal, dessa forma, espera-se que a significância do teste seja maior que 0,000 , para que se aceite a hipótese nula. Ao realizar o KS, constatou-se que a amostra de resíduos apresenta normalidade, já que a significância do teste foi 0,215, e, dessa forma, não se pode rejeitar a hipótese nula. 0 resultado do teste pode ser visto na Tabela 5.

Tabela 5. Teste Kolmogorov-Smirnov

\begin{tabular}{l|c|c}
\hline \multicolumn{2}{l|}{ Amostra Total } & $\mathbf{2 4 4}$ \\
\hline \multirow{2}{*}{ Diferenças mais extremas } & Absoluto & 0,068 \\
\cline { 2 - 3 } & Positivo & 0,068 \\
\cline { 2 - 3 } & Negativo & $-0,052$ \\
\hline Teste estático & 1,056 \\
\hline Sig. (2-sided test) & 0,215 \\
\hline
\end{tabular}

O último pressuposto da regressão a ser analisado foi a homoscedasticidade. Segundo Corrar et al. (2009), para isso, pode-se utilizar o teste de Pesarán-Pesarán ou o teste de White, contudo esse último não está disponível no software SPSS 20.o. Dessa forma, foi utilizado o teste de Pesarán-Pesarán, que consiste em regredir o quadrado dos resíduos padronizados $\left(Z R E^{2}\right)$ como função do quadrado dos valores estimados padronizados $\left(Z P R^{2}\right)$. A hipótese nula é que os resíduos são homoscedásticos, assim espera-se que a significância do teste seja acima de o,000. Ao realizar o Pesarán-Pesarán, o teste ANOVA apresentou significância de 0,318 , e, dessa forma, constata-se que o modelo não apresenta problemas de heteroscedasticidade. Tal resultado pode ser visto na Tabela 6 , a seguir.
Tabela 6. Teste ANOVA dos resíduos da regressão

\begin{tabular}{l|c|c|c|c|c|c}
\hline \multicolumn{7}{l|}{ ANOVA $^{a}$} \\
\multicolumn{2}{c|}{ Modelo } & $\begin{array}{c}\text { Soma do } \\
\text { quadrados }\end{array}$ & $\mathrm{Gl}$ & $\begin{array}{c}\text { Quadrado } \\
\text { médio }\end{array}$ & $\mathrm{F}$ & Sig. \\
\hline \multirow{2}{*}{1} & Regressão & 2,310 & 1 & 2,310 & 1,002 & $0,318^{\mathrm{b}}$ \\
\cline { 2 - 7 } & Resíduos & 558,038 & 242 & 2,306 & & \\
\cline { 2 - 7 } & Total & 560,348 & 243 & & & \\
\hline
\end{tabular}

a. Variável dependente: ZRE_2

b. Preditores: (Constant), ZPR_2

Conforme pode-se constatar, todos os testes utilizados para analisar os pressupostos da regressão foram satisfatórios, e, com isso, afirma-se a integridade dos testes de ajustamento e de significância do modelo. Com o objetivo de sintetizar os resultados da pesquisa, apresenta-se o Quadro 1, onde podem ser observadas quais hipóteses foram suportadas e quais não foram pelo modelo.

\section{Quadro 1. Síntese dos resultados}

\begin{tabular}{|c|c|c|}
\hline Hipóteses & Variáveis & Resultado \\
\hline \multirow[b]{2}{*}{$\begin{array}{l}\text { H1: Orientação } \\
\text { Socioambiental }\end{array}$} & $\begin{array}{l}\text { Ética nos negócios } \\
\text { (não significante) }\end{array}$ & \multirow[b]{2}{*}{$\begin{array}{l}\text { Suportada } \\
\text { parcialmente }\end{array}$} \\
\hline & $\begin{array}{l}\text { Governança } \\
\text { corporativa } \\
\text { (significante) }\end{array}$ & \\
\hline \multirow{3}{*}{$\begin{array}{l}\mathrm{H} 2 \text { : Engajamento com } \\
\text { stakeholders }\end{array}$} & $\begin{array}{l}\text { Sociedade e } \\
\text { comunidade } \\
\text { (significante) }\end{array}$ & \multirow{3}{*}{$\begin{array}{l}\text { Suportada } \\
\text { parcialmente }\end{array}$} \\
\hline & $\begin{array}{l}\text { Relacionamento social } \\
\text { com fornecedores } \\
\text { (não significante) }\end{array}$ & \\
\hline & $\begin{array}{l}\text { Política pública (não } \\
\text { significante) }\end{array}$ & \\
\hline \multirow[b]{2}{*}{$\begin{array}{l}\mathrm{H}_{3} \text { : Aperfeiçoamento } \\
\text { ambiental }\end{array}$} & $\begin{array}{l}\text { Operações } \\
\text { (significante) }\end{array}$ & \multirow[b]{2}{*}{$\begin{array}{l}\text { Não } \\
\text { suportada }\end{array}$} \\
\hline & $\begin{array}{l}\text { Relacionamento com } \\
\text { fornecedores na } \\
\text { cadeia de suprimentos } \\
\text { (significante) }\end{array}$ & \\
\hline $\mathrm{H}_{4}$ : Capital humano & $\begin{array}{l}\text { Tratamento dos } \\
\text { funcionários (não } \\
\text { significante) }\end{array}$ & $\begin{array}{l}\text { Não } \\
\text { suportada }\end{array}$ \\
\hline
\end{tabular}




\section{DISCUSSÃO E CONCLUSÕES}

Do construto orientação socioambiental, somente a variável governança corporativa pôde ser suportada, sendo inclusive a variável independente de maior influência sobre a ambiguidade causal. Estudos anteriores de Post et al. (2011) e Walls et al. (2012) já indicavam a importância da governança. Walls et al. (2012), a partir de pesquisa empírica, já tinham investigado a influência da governança sobre a estratégia ambiental, encontrando um efeito positivo quando os membros do conselho de administração tinham uma orientação voltada ao apoio de questões sociais e ambientais. Nessa linha, o presente estudo amplia a importância da governança, mostrando que esta também impacta de maneira positiva a criação de ambiguidade causal. A atuação do conselho envolve recursos difíceis de copiar, como conselheiros que são influenciados por suas diferentes experiências de vida e profissional, pela cultura da empresa e pelo modelo de atuação de uma indústria, que interagem formando uma capacidade única.

A ética nos negócios, outra variável do construto orientação socioambiental, surpreendeu pelo fato de não apresentar impacto sobre a ambiguidade causal. Conforme Husted e Allen (2000), no âmbito de uma visão utilitarista, a empresa poderia utilizar a ética para gerar vantagens, contudo, no longo prazo, uma estratégia baseada na ética pode tornar-se padrão em uma indústria. Ou seja, outras empresas podem adotar a ética como estratégia, tornando essa prática comum e não diferenciada. Assim, caso ocorra a padronização do comportamento ético, dificilmente uma empresa poderá gerar ambiguidade causal a partir desse recurso, pois, apesar de intangível, a percepção dos públicos de interesse (clientes, fornecedores, parceiros, governo etc.) poderá ser a de que todas as empresas utilizam o mesmo padrão, mesmo que, muitas vezes, seja colocado em prática de modos diferentes.

Por outro lado, essa padronização também pode ocorrer por meio da rigorosa aplicação da legislação. Em países da Europa e no Japão, por exemplo, há leis de combate à corrupção que exigem das empresas a criação de mecanismos para inibi-la, e contam com punições severas, o que leva à padronização de procedimentos, um típico exemplo de força isomórfica coercitiva.

No âmbito do construto engajamento com stakeholders, somente a variável sociedade/comunidade impactou a ambiguidade causal. Trabalhos anteriores como o de Bandeira-de-Mello, Marcon, e Alberton (2011) já tinham identificado a influência do stakeholder comunidade no desempenho das empresas. Nessa linha, o presente trabalho amplia a importância de se relacionar com essa importante parte interessada, já que tal relacionamento impacta positivamente a criação de ambiguidade causal. Corroborando os argumentos de Hart (1995), Sharma e Vredenburg
(1998), Sharma e Henriques (2005), Black (2006) e Molteni (2006), o processo de relacionamento entre a empresa e seus públicos de interesse é complexo, e tal característica torna difícil o entendimento e a imitação pelos concorrentes. Além disso, ao se relacionar com o público sociedade/comunidade, a empresa aprende e apreende competências que não são de sua especialidade, e que podem ajudar a gerar vantagens nos termos do negócio. Isso torna difícil o entendimento pelos concorrentes sobre onde a empresa adquiriu tais competências. Conforme Molteni (2006), a integração com os stakeholders e o entendimento de suas expectativas pode ajudar a empresa a encontrar soluções inovadoras a partir da RSAE, gerando vantagens para os negócios. Já as variáveis "relacionamento social com os fornecedores" e "relacionamento com o governo" (política pública) não impactaram a ambiguidade causal.

No âmbito do construto aperfeiçoamento ambiental, surpreendeu o fato de as variáveis não impactarem positivamente a criação de ambiguidade causal, mas sim negativamente. Diversos autores argumentaram que questões como a prevenção da poluição (Aragón-Correa \& Sharma, 2003; Hart, 1995; Russo \& Fouts, 1997; Sharma \& Vredenburg, 1998), a colaboração na cadeia de suprimentos (Klassen \& Vachon, 2003) e a participação em redes de suprimentos (Walls et al., 2011) são atividades complexas e que exigem esforços na coordenação de equipes envolvidas, assimilação de outras culturas organizacionais, uso de tecnologia, entre a utilização e integração de outros recursos intangiveis. Contudo, pode-se observar também que vem ocorrendo um processo de padronização no que diz respeito às questões operacionais voltadas para o meio ambiente. A popularização dos sistemas de gestão ambiental baseadas na norma ISO 14001, por exemplo, tem produzido uma difusão de práticas operacionais, e, ainda, necessariamente em conformidade com a legislação do país e dos países importadores, e essas leis são mutuamente influenciadas ou baseadas em acordos intergovernamentais multilaterais globais ou regionais. Assim, normas e acordos internacionais têm influenciado, em cadeia, certificações e práticas operacionais que tratam de questões ambientais. Esta pode ser a principal explicação para o impacto do construto aperfeiçoamento ambiental sobre a ambiguidade causal, já que o sinal negativo representa que quanto maior o aperfeiçoamento ambiental, menor a ambiguidade causal.

No âmbito do construto capital humano, também surpreendeu negativamente o fato de não impactar a ambiguidade causal, já que a expectativa era de que essa dimensão influenciasse em alto nível a variável dependente. Contudo, uma explicação razoável foi encontrada para justificar esse resultado. Ao observar com maior minúcia os indicadores utilizados pela Sustainalytics, pode-se observar que são basicamente voltados para questões 
relacionadas com os direitos humanos do trabalho, conforme a Organização Internacional do Trabalho (ITL) e que foram incorporadas nas legislações dos países considerados na amostra, tais como liberdade sindical, discriminação, diversidade, acordos coletivos, incidentes de saúde e segurança, e fatalidades. A priori, tais indicadores não são a melhor representação da estratégia socioambiental baseada no capital humano. Os argumentos de Hart (1995), Hart e Milstein (2003), McWilliams e Siegel (2011), Surroca et al. (2010), entre outros, utilizados para derivar a hipótese de que a estratégia socioambiental baseada no capital humano impacta significativamente de maneira positiva na ambiguidade causal, levam em consideração aspectos como retenção de talentos, aumento de produtividade, motivação dos funcionários, desenvolvimento das pessoas, cultura organizacional, entre outros. Os indicadores da Sustainalytics provalmente não captam esse tipo de indicador.

Em conclusão, a utilização da ambiguidade causal como variável dependente é fato novo e relevante nos estudos da área de RBV, bem como sua relação com a estratégia socioambiental. Desse modo, pode-se afirmar que os resultados desta pesquisa exploratória têm potencial para ampliar as discussões na intersecção dos estudos de RBV e RSAE. $O$ fato de as hipóteses não terem sido suportadas totalmente, ou não suportadas, faz parte do processo de pesquisa exploratória, quando são pesquisados novos assuntos e novas relações teóricas e empíricas.

\section{REFERÊNCIAS}

Ambrosini, V., \& Bowman, C. (2005). Reducing causal ambiguity to facilitate strategic learning. Management Learning, 36(4), 493-512. doi: $10.1177 / 1350507605058142$

Aragón-Correa, J. A., \& Sharma, S. (2003). A contingent resource-based view of proactive corporate environmental strategy. Academy of Management Review, 28(1), 71-88.

Atkinson, A. A., Waterhouse, J. H., \& Wells, R. B. (1997). A stakeholder approach to strategic performance measurement. Sloan Management Review. Recuperado de http://sloanreview.mit.edu/

Bandeira-de-Mello, R., Marcon, R., \& Alberton, A. (2011). Performance effects of stakeholder interaction in emerging economies: Evidence from Brazil. BAR-Brazilian Administration Review, 8(3), 329-350. doi:10.1590/s1807-76922011000300007

Bansal, P., \& Roth, K. (2000). Why companies go green: A model of ecological responsiveness. Academy of Management Journal, 43(4), 717-736.

Barney, J. (1986). Organizational culture: Can it be a source of sustained competitive advantage? Academy of Management Review, 11(3), 656-665.

Barney, J. (1991). Firm resources and sustained competitive advantage. Journal of Management, 17(1), 99-120. doi:10.1177/014920639101700108
Becker, B., \& Gerhart, B. (1996). The impact of human resource management on organizational performance: Progress and prospects. Academy of Management Journal, 39(4), 779-801.

Black, L. D. (2006). Corporate social responsibility as capability: The case of BHP Billiton. Journal of Corporate Citizenship, 23, 25-39.

Branco, M. C., \& Rodrigues, L. L. (2006). Corporate social responsibility and resource-based perspectives. Journal of Business Ethics, 69(2), 111-132. doi:10.1007/s10551-006-9071-z

Burke, L., \& Logsdon, J. M. (1996). How corporate social responsibility pays off. Long Range Planning, 29(4), 495-502. doi:10.1016/00246301(96)00041-6

Cooper, D. R., \& Schindler, P. S. (2011). Métodos de pesquisa em administração. Porto Alegre: Bookman.

Corrar, L. J., Paulo, E., \& Dias-Filho, J. M. (2009). Análise multivariada: Para cursos de administração, ciências contábeis e economia. São Paulo: Atlas.

Dierickx, I., \& Cool, K. (1989). Asset stock accumulation and sustainability of competitive advantage. Management Science, 35(12), 1504-1514. doi:10.1287/mnsc.35.12.1504

Donaldson, T., \& Preston, L. (1995). The stakeholder theory of the corporation: Concepts, evidence and implications. Academy of Management Review, 20(1), 65-91.

Dyer, J. H., \& Singh, H. (1998). The relational view: Cooperative strategy and sources of interorganizational competitive advantage. Academy of Management Review, 23(4), 660-679. doi:10.5465/ amr.1998.1255632

González-Alvarez, N., \& Nieto-Antolín, M. (2005). Protection and internal transfer of technological competencies: The role of causal ambiguity. Industrial Management; Data Systems, 105(7), 841-856.

Griffin, J. J., \& Mahon, J. F. (1997). The corporate social performance and corporate financial performance debate: Twenty-five years of incomparable research. Business and Society, 36(1), 5-31. doi:10.1177/000765039703600102

Hair, J. F., Jr., Babin, B., Money, A. H., \& Samouel, P. (2005). Fundamentos de métodos de pesquisa em administração. Porto Alegre: Bookman.

Hair, J. F., Jr., Black, W. C., Babin, B. J., Anderson, R. E., \& Tatham, R. L. (2009). Análise multivariada de dados (6a ed.). Porto Alegre: Bookman.

Hanna, M. D., Newman, W. R., \& Johnson, P. (2000). Linking operational and environmental improvement through employee involvement. International Journal of Operations \& Production Management, 20(2), 148-165. doi:10.1108/01443570010304233

Hart, S. L. (1995). A natural-resource-based view of the firm. Academy of Management Review, 20(4), 986-1014.

Hart, S. L., \& Milstein, M. B. (2003). Creating sustainable value. Academy of Management Executive, 17(2), 56-69.

Hart, S. L., \& Dowell, G. (2011). A natural resource-based view of the firm: Fifteen years after. Journal of Management, 37(5), 1464-1479. doi:10.1177/0149206310390219

Huselid, M. A. (1995). The impact of human resource management practices on turnover, productivity, and corporate financial performance. Academy of Management Journal, 38(3), 635-672. doi:10.2307/256741

Husted, B. W. (2003). Governance choices for corporate social responsibility: To contribute, collaborate or internalize? Long Range Planning, 36(5), 481-498. doi:10.1016/S0024-6301(03)00115-8 
Husted, B. W., \& Allen, D. B. (2000). Is it ethical to use ethics as strategy? Journal of Business Ethics, 27(1-2), 21-31. doi:10.1023/A:1006422704548

Husted, B. W., \& Allen, D. B. (2007a). Corporate social strategy in multinational enterprises: Antecedents and value creation. Journal of Business Ethics, 74(4), 345-361. doi:10.1007/s10551-007-9511-4

Husted, B. W., \& Allen, D. B. (2007b). Strategic corporate social responsibility and value creation among large firm: Lessons from the Spanish experience. Long Range Planning, 40(6), 594-610. doi:10.1016/j.Irp.2007.07.001

Husted, B. W., \& Allen, D. B. (2009). Strategic corporate social responsibility and value creation: A study of multinational enterprises in Mexico. Management International Review, 49(6), 781-799. doi:10.1007/s11575-009-0016-5

Kayo, E. K., \& Famá, R. (2004). A estrutura de capital e o risco das empresas tangível-intensivas e intangível-intensivas. RAUSP-Revista de Administração da Universidade de São Paulo), 39(2), 164-176.

King, A. W. (2007). Disentangling interfirm and intrafirm causal ambiguity: A conceptual model of causal ambiguity and sustainable competitive advantage. Academy of Management Review, 32 (1), 156178. doi:10.2307/20159286

King, A. W., \& Zeithaml, C. P. (2001). Competencies and firm performance: Examining the causal ambiguity paradox. Strategic Management Journal, 22(1), 75-99. doi:10.1002/1097-0266(200101)22:1<75::aidsmj145’3.0.c0;2-i

Kitazawa, S., \& Sarkis, J. (2000). The relationship between ISO 14001 and continuous source reduction programs. International Journal of Operations \& Production Management, 20(2), 225-248. doi:10.1108/01443570010304279

Klassen, R. D., \& Vachon, S. (2003). Collaboration and evaluation in the supply chain: The impact on plant-level environmental investment. Production and Operations Management, 12(3), 336352. doi:10.1111/j.1937-5956.2003.tboo207.x

Kleindorfer, P. R., Singhal, K., \& Wassenhove, L. N. (2005). Sustainable operations management. Production and Operations Management, 14(4), 482-492. doi:10.1111/j.1937-5956.2005.tboo235.x

Linton, J. D., \& Jayaraman, V. (2005). A framework for identifying differences and similarities in the managerial competencies associated with different modes of product life extension. International Journal of Production Research, 43(9), 1807-1829. doi: $10.1080 / 13528160512331326440$

Linton, J. D., Klassen, R., \& Jayaraman, V. (2007). Sustainable supply chains: An introduction. Journal of Operations Management, 25(6), 1075-1082. doi:10.1016/j.jom.2007.01.012

Mahoney, J. T., \& Pandian, J. R. (1992). The resource-based view within the conversation of strategic management. Strategic Management Journal, 13(5), 363-380. doi:10.1002/smj.4250130505

McWilliams, A., \& Siegel, D. S. (2001). Corporate social responsibility: A theory of the firm perspective. Academy of Management Review, 26(1), 117-127.

McWilliams, A., \& Siegel, D. S. (2011). Creating and capturing value: Strategic corporate social responsibility, resource-based theory, and sustainable competitive advantage. Journal of Management, 37(5), 1480-1495. doi:10.1177/0149206310385696

Melnyk, S. A., Sroufe, R. P., \& Calantone, R. (2003). Assessing the impact of environmental management systems on corporate and environmental performance. Journal of Operations Management, 21(3), 329-351. doi:10.1016/So272-6963(02)00109-2

Molteni, M. (2006). The social-competitive innovation pyramid. Corporate Governance: The International Journal of Business in Society, 6(4), 516-526. doi:10.1108/14720700610689612

Mosakowski, E. (1997). Strategy making under causal ambiguity: Conceptual issues and empirical evidence. Organization Science, $8(4), 414-442$.

Nelson, R., \& Winter, S. (1982). An evolutionary theory of economic change. Cambridge, MA: Belknap Press.

Nyborg, K., \& Brekke, K. A. (2004). Moral hazard and moral motivation: Corporate social responsibility as labor market screening. Working paper no. 25/2004, University of Oslo Economics. Retrieved from http://ssrn.com/abstract $=645741$.

Oliveira, A. M. B., \& Oliveira, A. J. (2011). Gestão de recursos humanos: Uma metanálise de seus efeitos sobre desempenho organizacional. RAC-Revista de Administração Contemporânea, 15(4), 650-669. doi:10.1590/s1415-65552011000400006

Peteraf, M. A. (1993). The cornerstones of competitive advantage: A resource-based view. Strategic Management Journal, 14(3), 179-191. doi:10.1002/smj.4250140303

Pfeffer, J., \& Veiga, J. F. (1999). Putting people first for organizational success. Academy of Management Executive, 13(2), 37-48.

Polanyi, M. (1967). The tacit dimension. Garden City, NY: Anchor.

Porter, M. E., \& Kramer, M. (2002). The competitive advantage of corporate philanthropy. Harvard Business Review. Recuperado de https://hbr.org/.

Porter, M. E., \& Kramer, M. (2006). Strategy and society: The link between competitive advantage and corporate social responsibility. Harvard Business Review. Recuperado de https://hbr.org.

Porter, M. E., \& Kramer, M. (2011). Creating shared value: How to reinvent capitalism and unleash a wave of innovation and growth. Harvard Business Review. Recuperado de https://hbr.org.

Porter, M. E., \& Van der Linde, C. (1995). Green and competitive: Ending the stalemate. Harvard Business Review. Recuperado de https://hbr. org.

Post, C., Rahman, N., \& Rubow, E. (2011). Green governance: Boards of directors' composition and environmental corporate social responsibility. Business and Society, 50(1), 189-223. doi:10.1177/0007650310394642

Powell, T. C. (2001). Competitive advantage: Logical and philosophical considerations. Strategic Management Journal, 22(9), 875-888. doi:10.1002/smj.173

Powell, T. C., Lovallo, D., \& Caringal, C. (2006). Causal ambiguity, management perception, and firm performance. Academy of Management Review, 31(1), 175-196. doi:10.2307/20159191

Reed, R., \& DeFillippi, R. J. (1990). Causal ambiguity, barriers to imitation, and sustainable competitive advantage. Academy of Management Review, 15(1), 88-102.

Rumelt, R. (1984). Toward a strategic theory of the firm. In R. Lamb (ed.), Competitive Strategic Management, 556-570. Englewood Cliffs, NJ: Prentice Hall.

Russo, M. V., \& Fouts, P. A. (1997). A resource-based perspective on corporate environmental performance and profitability. Academy of Management Journal, 40(3), 534-559. 
Sarkis, J. (1995). Supply chain management and environmentally conscious design and manufacturing. International Journal of Environmentally Conscious Design and Manufacturing, 4(2), 43-52.

Sharfman, M. P., Pinkston, T. S., \& Sigerstad, T. D. (2000). The effects of managerial values on social issues evaluation: An empirical examination. Business \& Society, 39(2), 144-182. doi:10.1177/000765030003900203

Sharma, S., Pablo, A. L., \& Vredenburg, H. (1999). Corporate environmental responsiveness strategies: The importance of issue interpretation and organizational context. Journal of Applied Behavioral Science, 35(1), 87-108. doi:10.1177/0021886399351008

Sharma, S. (2000). Managerial interpretations and organizational context as predictors of corporate choice of environmental strategy. Academy of Management Journal, 43(4), 681-697.

Sharma, S., \& Henriques, I. (2005). Stakeholder influences on sustainability practices in the Canadian forest products industry. Strategic Management Journal, 26(2), 159-180. doi:10.1002/smj.439

Sharma, S., \& Vredenburg, H. (1998). Proactive corporate environmental strategy and the development of competitively valuable organizational capabilities. Strategic Management Journal, 19(8), 729-753. doi:10.1002/(sici)1097-0266(199808)19:8〈729::aid-smj967〉3.0. $\mathrm{CO} ; 2-4$
Shrivastava, P. (1995). Environmental technologies and competitive advantage. Strategic Management Journal, 16(S1), 183-200. doi:10.1002/smj.4250160923

Simonin, B. L. (1999). Ambiguity and the process of knowledge transfer in strategic alliances. Strategic Management Journal, 20(7), 595623. doi:10.1002/(sici)1097-0266(199907)20:7〈595::aid-smj47’3.0. Co;2-5

Sroufe, R. (2003). Effects of environmental management systems on environmental management practices and operations. Production and Operations Management, 12(3), 416-431. doi:10.1111/j.1937-5956.2003.tboo212.x

Surroca, J., Tribó, J. A., \& Waddock, S. (2010). Corporate responsibility and financial performance: The role of intangible resources. Strategic Management Journal, 31(5), 463-490. doi:10.1002/smj.820

Walls, J. L., Berrone, P., \& Phan, P. H. (2012). Corporate governance and environmental performance: Is there really a link? Strategic Management Journal, 33(8), 885-913. doi:10.1002/smj.1952

Walls, J. L., Phan, P. H., \& Berrone, P. (2011). Measuring environmental strategy: Construct development, reliability, and validity. Business and Society, 50(1), 71-115. doi:10.1177/0007650310394427

Williamson, O. E. (1985). The economic institutions of capitalism. New York: Free Press 